\title{
EDIFİCATE
}

I Congreso de Escuelas de Edificación y Arquitectura Técnica de España

València, 4 y 5 de noviembre de 2021

Escuela Técnica Superior de Ingeniería de Edificación

Universitat Politècnica de València

Doi: https://doi.org/10.4995/EDIFICATE2021.2021.13587

\section{Experiencias en docencia en inglés}

\section{English teaching experiences}

\section{María-Isabel Giner-García ${ }^{a}$, Ángeles Rodrigo-Molina ${ }^{b}$}

${ }^{a}$ Escuela Técnica Superior de Ingeniería de Edificación, Universitat Politècnica de València, Camino de Vera s/n, 46022, Valencia (España). magigar@csa.upv.es.

${ }^{\mathrm{b}}$ Escuela Técnica Superior de Ingeniería del Diseño, Universitat Politècnica de València, Camino de Vera s/n, 46022, Valencia (España). arodrigo@ega.upv.es

\begin{abstract}
In the 21st century University, and increasingly so, the knowledge and mastery of English as a first foreign language is necessary for the integral education of students, and has become essential for integration into the labour market. For this reason, the internationalisation strategies or language policy plans of the vast majority of Spanish universities include the introduction of teaching in English in their degree programmes, enabling them to attract international students as well.

In this line, the School of Building Engineering of the Polytechnic University of Valencia reached the point where English teaching was implemented in the entire Bachelor's Degree in Technical Architecture in the 2016-2017 academic year, although currently only a few subjects remain in the 2nd year. As a result, we became personally involved as lecturers in a total of 6 subjects, both in the Building Techniques and Technology module and in the Urban Management and Applied Economics module, as well as in the Bachelor's Degree Thesis.
\end{abstract}

The aim of this paper is to present the balance of the English teaching experiences in the aforementioned subjects of the Degree in Technical Architecture of the School of Building Engineering of the Polytechnic University of Valencia.

Keywords: Bachelor's Degree Thesis; Budget and Economic Control topics; Building Techniques and Technology module; Construction topics; English teaching; Experts, Appraisals, and Valuations topics; Urban Management and Applied Economics module. 


\section{Resumen}

En la Universidad del siglo XXI, y cada vez más en auge, el conocimiento y el dominio del inglés como primera lengua extranjera es necesario en la formación integral del alumno, y se ha tornado imprescindible para la inserción en el mundo laboral. De ahí que las estrategias de internacionalización o planes de política lingüística de la gran mayoría de universidades españolas pase por introducir la docencia en inglés en sus titulaciones, permitiendo además atraer al alumno internacional.

En esta línea, la Escuela Técnica Superior de Ingeniería de Edificación de la Universitat Politécnica de València llegó a tener implantada la docencia en inglés en la totalidad del Grado en Arquitectura Técnica en el curso académico 2016-2017, aunque en la actualidad sólo quedan algunas asignaturas de $2^{\circ}$ curso. Como resultado se llegó a tener una implicación personal como docente en un total de 6 asignaturas, tanto del módulo de Técnicas y Tecnología de la Edificación, como del de Gestión Urbanística y Economía Aplicada, así como en el Trabajo Final de Grado.

El objetivo del presente trabajo, es dar a conocer el balance sobre las experiencias en docencia en inglés en el Grado de Arquitectura Técnica de la ETSIE de la UPV.

Palabras clave: docencia en inglés; materias de Construcción; materias de Presupuestos y Control Económico; materias de Peritaciones, Tasaciones y Valoraciones; módulo de Gestión Urbanística y Economía Aplicada; módulo de Técnicas y Tecnología de la Edificación; Trabajo Final de Grado. 


\section{Introducción}

En la Universidad del siglo XXI, y cada vez más en auge, el conocimiento y el dominio del inglés como primera lengua extranjera es necesario en la formación integral del alumno, y cada vez más resulta requisito impresciondible para la inserción en el mundo laboral.

Las estrategias de internacionalización o planes de política lingüística de la gran mayoría de universidades españolas pasa por introducir la docencia en inglés en sus titulaciones, permitiendo además atraer al alumno internacional.

Algunas universidades como la Universitat Politècnica de València (UPV) requieren a su estudiantado la acreditación del conocimiento de un idioma extranjero con un nivel mínimo de B2 para la obtención del título.

\section{Objetivos}

El objetivo es dar a conocer el balance sobre las experiencias en docencia en inglés en el Grado de Arquitectura Técnica de la Escuela Técnica Superior de Ingeniería de Edificación (ETSIE) de la UPV.

\section{Desarrollo de la Innovación}

\subsection{Importancia de conocimiento del inglés como idioma extranjero}

El conocimiento del inglés como idioma extranjero tiene importancia no sólo desde el punto de vista curricular, sino también en el no curricular.

Por normativa UPV (UPV Servei d'Alumnat, s.f.), todos los alumnos deben acreditar el conocimiento de una lengua extranjera, preferentemente el inglés, con un nivel correspondiente al B2 de los niveles comunes de referencia fijados por el "Marco Común Europeo de Referencia para las lenguas: aprendizaje, enseñanza, evaluación".

Es recomendable también la formación en lengua inglesa para el caso en que se desee participar en programas de intercambio internacional, debido a la necesidad de acreditar un nivel mínimo de B2 de inglés.

Según se desprende de la Convocatoria y Bases Específicas de Programa Erasmus+Estudios publicada como Anexo I por la OPII -Oficina de Programas Internacionales de Intercambio- de la UPV (UPV Oficina de Programes Internacionals d'Intercanvi, s.f.), y específicamente para las titulaciones de Grado en Arquitectura Técnica y Máster en Edificación cursadas en la ETSIE (ETSIE, s.f.-a), la docencia en inglés representa un $5 \%$ de la puntuación total. Así mismo, permite la obtención de puntos adicionales para optar a las mencionadas becas Erasmus+, obteniendo 2 puntos por cada asignatura técnica cursada (y superada) en inglés durante los estudios de grado y hasta un máximo de 10 puntos, frente a los 4 puntos que se alcanzarian según la tabla de equivalencias con respecto al Marco Europeo de Referencia. 
A tener en cuenta la doble ventaja que, desde el punto de vista curricular, presenta la oferta realizada por la ETSIE de recibir docencia en inglés en el Grado en Arquitectura Técnica. Por un lado, permite al alumnado la obtención de créditos para convalidación o reconocimiento académico del nivel B2 de lengua extranjera necesario para la obtención del título. Y por otro, la obtención de puntos adicionales para el acceso a becas de intercambio internacional (ETSIE, s.f.-b).

Con la docencia en inglés, se adquieren y desarrollan competencias que identifican elementos compartidos comunes a cualquier titulación, como la competencia transversal número 009, en la que se considera la apreciación de la diversidad y multiculturalidad. Habilidad para trabajar en un contexto internacional. Capacidad para adaptarse a nuevas situaciones. Asímismo, también se alcanzan competencias específicas, como la número 062, en la que se destaca la capacidad para comunicarse oralmente en un segundo idioma en un entorno profesional o académico...Competencias estas, marcadas por la Memoria de Solicitud de Verificación del Título de Mayo de 2010, especificadas en el Libro Blanco, y debiendo ser garantizada su adquisición por el Plan de Estudios.

\subsection{Situación de docencia en inglés en la Escuela Técnica Superior de Ingeniería de Edificación de la UPV}

La línea de docencia en inglés impartida en la titulación de Grado en Arquitectura Técnica de la ETSIE de la UPV se inició en el curso académico 2013-2014, implantándose de forma progresiva anualmente en cada uno de los cursos. De tal forma que, en el curso académico 2016-2017 la línea en inglés ya se impartía completamente en todo el Grado. Sin embargo, por decisiones políticas, a partir del curso 2019-2020 la mencionada línea empezó a desaparecer, iniciándose en las asignaturas de $4^{\circ}$ curso, y continuando con las asignaturas de $1^{\circ}$ y $3^{\circ}$ curso en el curso académico 2020-2021. En la actualidad únicamente queda docencia en inglés en algunas asignaturas de segundo curso (Instalaciones I y II, Materiales III, Construcción II, Construcción III, y Dibujo Arquitectónico II) (ETSIE, s.f.-c).

La docencia en inglés ha formado parte también del programa de movilidad internacional que oferta el Centro para sus estudiantes. Este programa de movilidad permite a los alumnos cursar asignaturas o desarrollar el Trabajo Final de Grado, pudiendo realizarse estancias en distintas universidades (UPV Titulaciones, s.f.) de algunos países europeos como Alemania, Dinamarca, Finlandia, Francia, Gales, Holanda, Hungría, Inglaterra, Italia, Lituania, Polonia, Portugal, República Checa, y Suecia, asícomo países de otros continentes como Cuba, Chile, China, etc.

La Escuela también ha promovido la matriculación de asignaturas con docencia en inglés, haciendo excepciones a la normativa de progreso y permanencia de la UPV, permitiendo el acceso a dichas asignaturas de cursos superiores desde el primer curso.

\subsection{Implicación personal en docencia en inglés}

El antecedente a la implicación docente se fraguó durante la época de estudiante, gracias a resultar beneficiada por una beca del Programa de movilidad de estudiantes ERASMUS 
(European Exchange Programme) en el curso 1996-1997, por un periodo de 6 meses para la realización del Proyecto Final de Carrera en HORSENS POLYTECHNIC (Denmark).

La experiencia de recibir como estudiante docencia en inglés resultó ser dura, pero muy positiva y enriquecedora, no sólo desde el punto de vista curricular sino también personal. La movilidad a otro país, con una cultura completamente diferente, y la convivencia con personas no sólo del país de destino sino también de otros países, aportaron un valor extra a la educación académica y evolución personal.

Desde la perspectiva curricular, aprender y desarrollar formas diferentes de entender la profesión (incluso, en ciertos aspectos, mucho más avanzados), ampliaron los conocimientos adquiridos durante la formación académica en el país de origen, que incluso se han podido aplicar durante el ejercicio profesional. La hicieron especialmente dura la combinación de varios factores como: el uso de una normativa diferente y específica del país de destino; un desarrollo tecnológico completamente distinto al estudiado durante la carrera; y el idioma. Es necesario reseñar que esta dificultad se pudo resistir y sobrellevar gracias a la ayuda y apoyo prestado tanto por el personal docente como por los compañeros. También mencionar la importancia y papel relevante que tuvo la existencia en inglés tanto de la normativa como de la bibliografía a consultar.

\section{Resultados}

\subsection{Implicación en docencia en inglés}

La implicación docente como profesora inició durante el curso académico 2005-2006, casualmente con la misma asignatura con la que finalizó la etapa de estudiante, y con el mismo centro de destino. Esto es, con la asignatura de Trabajo Final de Grado (en plan anterior Proyecto Final de Carrera), pero en este caso como tutora académica del alumnado de intercambio entre ambas escuelas _ETSIE_UNIVERSITAT POLITĖCNICA DE VALĖNCIA (España) y HORSENS POLYTECHNIC (Denmark)-.

La tabla 1 muestra un resumen de la implicación personal en docencia en inglés tanto como tutora académica como profesora. Tal y como se observa, a partir del curso 2015-2016 la implicación docente ha sido mayor, debido a la implantación de la línea de inglés en todo el Grado en Arquitectura Técnica, perdurando en el tiempo hasta la fecha. La docencia se ha llevado a cabo en distintas asignaturas, todas ellas de carácter troncal/obligatorio, y con una duración diferente en cada una de ellas, principalmente en el Grado en Arquitectura Técnica de la ETSIE, aunque también en el último curso académico ha implicado al Grado en Fundamentos de Arquitectura de la ETSA. 
Tabla 1. Resumen de la implicación personal en docencia en inglés tanto como tutora académica como profesora

\begin{tabular}{|c|c|c|c|c|c|}
\hline Asignatura & Curso & Semestre & Créditos & Tipo de materia & $\begin{array}{c}\text { Cursos } \\
\text { impartidos }\end{array}$ \\
\hline \multicolumn{6}{|l|}{$\begin{array}{l}\text { Grado en Arquitectura } \\
\text { Técnica }\end{array}$} \\
\hline Trabajo Final Grado & $4^{\circ}$ & 2 & 12 & $\begin{array}{l}\text { Proyecto Fin de } \\
\text { Grado }\end{array}$ & $\begin{array}{c}\text { Desde } \\
2005-2006 \\
\text { Hasta la } \\
\text { actualidad }\end{array}$ \\
\hline Construcción IV & $3^{\circ}$ & 1 & 4,5 & Construcción & $\begin{array}{l}2015-2016 \\
2016-2017\end{array}$ \\
\hline Construcción V & $3^{\circ}$ & 2 & 6 & Construcción & $\begin{array}{l}2018-2019 \\
2019-2020\end{array}$ \\
\hline Construcciones Históricas & $3^{\circ}$ & 1 & 4,5 & Construcción & $2018-2019$ \\
\hline $\begin{array}{l}\text { Técnicas de Gestión } \\
\text { Presupuestaria }\end{array}$ & $3^{\circ}$ & 2 & 6 & $\begin{array}{l}\text { Presupuestos y } \\
\text { Control } \\
\text { Económico }\end{array}$ & $\begin{array}{l}2018-2019 \\
2019-2020\end{array}$ \\
\hline $\begin{array}{l}\text { Tasaciones, Peritaciones y } \\
\text { Valoraciones }\end{array}$ & $4^{\circ}$ & 1 & 4,5 & $\begin{array}{l}\text { Peritaciones, } \\
\text { Tasaciones y } \\
\text { Valoraciones }\end{array}$ & $\begin{array}{l}2016-2017 \\
2017-2018 \\
2018-2019 \\
\end{array}$ \\
\hline \multicolumn{6}{|l|}{$\begin{array}{l}\text { Grado en Fundamentos de } \\
\text { la Arquitectura }\end{array}$} \\
\hline Introducción a la Construcción & $1^{\circ}$ & 1 & 4,5 & Construcción & $2020-2021$ \\
\hline
\end{tabular}

\subsection{Perfil del alumnado}

El perfil de acceso del alumnado al Grado en Arquitectura Técnica en la línea en inglés es tanto español como extranjero. El estudiante extranjero procede tanto de Europa como de otros continentes, especialmente Asia. Los países mayoritarios son: Italia, Francia, Bélgica, Holanda, Alemania, Dinamarca, Suecia, Noruega, Finlandia, Polonia, Lituania, Hungría, Ucrania, República Checa, Eslovaquia, y China. Y los minoritarios han sido: Turquía, y Australia.

Sin embargo, la procedencia del alumnado extranjero para el Grado en Fundamentos en Arquitectura ha sido mayoritariamente de los países del Norte de África (en especial Marruecos y Argelia), y muy puntual países del Sur de América (como Venezuela, y Curaçao), y de Europa (Italia y Ucrania).

La demanda de la docencia en inglés, en el Grado en Arquitectura Técnica, históricamente ha sido moderada (en torno a los 25 alumnos máximo en el grupo entre españoles y extranjeros), y variable dependiendo del curso académico, semestre y asignaturas. Sin embargo, esta demanda, contrasta con la asignatura de Grado en Fundamentos de Arquitectura, es justamente a la inversa, tanto en procedencia del alumnado como en número total de alumnos en el grupo, llegando incluso a duplicarla. 
Tabla 2. Procedencia del alumnado demandante de docencia en inglés

\begin{tabular}{|c|c|c|c|c|c|c|c|c|c|c|c|c|}
\hline \multirow{3}{*}{$\begin{array}{l}\text { Asignaturas } \\
\text { Grado en } \\
\text { Arquitectura } \\
\text { Técnica }\end{array}$} & \multicolumn{2}{|c|}{$2015-2016$} & \multicolumn{2}{|c|}{$2016-2017$} & \multicolumn{2}{|c|}{$2017-2018$} & \multicolumn{2}{|c|}{$2018-2019$} & \multicolumn{2}{|c|}{$2019-2020$} & \multicolumn{2}{|c|}{$2020-2021$} \\
\hline & Esp. & Otros & Esp. & Otros & Esp. & Otros & Esp. & Otros & Esp. & Otros & Esp. & Otros \\
\hline & & & & & & & & & & & & \\
\hline Const. IV & $100 \%$ & $0 \%$ & $23 \%$ & $77 \%$ & $11 \%$ & $89 \%$ & \begin{tabular}{|l|}
$21 \%$ \\
\end{tabular} & $79 \%$ & $0 \%$ & $100 \%$ & & \\
\hline Const. V & $80 \%$ & $20 \%$ & $44 \%$ & $56 \%$ & $23 \%$ & $77 \%$ & $80 \%$ & $20 \%$ & $0 \%$ & $100 \%$ & & \\
\hline Const. Históricas & & & & & & & $10 \%$ & $90 \%$ & & & & \\
\hline TGP & & & & & & & $17 \%$ & $83 \%$ & $0 \%$ & $100 \%$ & & \\
\hline Tasac., Val. y Perit. & & & $37 \%$ & $63 \%$ & $16 \%$ & $84 \%$ & $0 \%$ & $100 \%$ & & & & \\
\hline $\begin{array}{l}\text { Fundamentos } \\
\text { en Arquitectura }\end{array}$ & & & & & & & & & & & & \\
\hline ICO & & & & & & & & & $60 \%$ & $40 \%$ & $56 \%$ & $44 \%$ \\
\hline
\end{tabular}

Habría que preguntarse el porqué de la baja demanda de alumnos españoles que hay en el Grado en Arquitectura Técnica frente a la que se produce en el Grado en Fundamentos en Arquitectura. Las razones podrían atender a varios factores: el nivel de inglés del alumnado; el nivel de inglés del profesorado; la escasez de bibliografía y material de estudio de las asignaturas; y el esfuerzo adicional que supone estudiar materias en lenguas diferentes a la lengua materna/nativa.

La heterogeneidad y variedad de procedencia del alumnado, no sólo se hacía patente en las nacionalidades, sino también en los Grados, con la consiguiente dispersión en cuanto a conocimientos previos de las materias objeto de estudio.

\subsection{Recursos}

Una característica común y generalizada para todas las asignaturas ha sido la inexistencia de material docente propio en inglés, y en la mayoría de los casos incluso escasa bibliografía en inglés para poder consultar. En otros casos, teniendo en cuenta la selección y estructuración de las unidades didácticas de las asignaturas hubiera sido posible facilitar al alumno bibliografía, sin embargo, los temas eran tan variados que la bibliografía a consultar hubiese tenido que ser de igual modo muy diversa, y se hubiera causado la dispersión del alumno.

Con respecto al material docente de las asignaturas impartidas, se daban casos de diversa índole: asignaturas cuyo material docente en español era escaso o nulo, por lo que ha sido necesaria una preparación de material previa a su traducción; asignaturas que contaban con presentaciones en power point en español, por lo que se ha procedido a traducir dichas presentaciones; $y$, asignaturas que ya contaban con presentaciones traducidas por otros profesores, procediendo en este caso a su revisión.

La traducción académica especializada ha sido propia en la mayoría de los casos. Unicamente se ha contado con una colaboración puntual en una de las asignaturas por otro 
profesor de la asignatura. Así mismo, en otra asignatura ha sido necesario recurrir a la contratación externa para la traducción de parte de la materia.

Como se ha mencionado, las traducciones de español a inglés en general han sido propias, por lo que se han realizado sin estar sometidas a control de calidad en lo referente a la revisión terminológica y gramatical. Es decir, no ha habido traductores/revisores nativos con formación en la especialidad o área de conocimiento, y por supuesto sin experiencia en la traducción, en particular la especializada.

A pesar de no haber contado en general con ayuda o apoyo para la traducción, se hace necesario mencionar que puntualmente y durante cierto tiempo, el Centro de Lenguas de la UPV ha puesto a disposición de los profesores con docencia en inglés sesiones para la revisión del material docente. Las sesiones eran individuales de una hora semanal o cada dos semanas, durante las cuales se repasaban las diapositivas que el profesor ya había confeccionado en inglés, y se resolvían dudas sobre vocabulario, pronunciación, etc. No obstante, debido a la gran cantidad de material docente que supervisar, el tiempo dedicado a ello resultó ser insuficiente. También destacar que aunque los revisores eran nativos no contaban con formación especializada en el área de conocimiento, lo cual representaba un obstáculo a la hora de aportar más rigor a la terminología específica.

\subsection{Resultados académicos del alumnado}

A modo de ejemplo, se muestran los resultados académicos obtenidos en el curso 20182019 para dos de las asignaturas, representativas a su vez de cada uno de los módulos en los que se ha impartido docencia: Construcciones Históricas (Tabla 3) del módulo de Técnicas y Tecnología de la Edificación, y Técnicas de Gestión Presupuestaria (Tabla 4) del módulo de Gestión Urbanística y Economía Aplicada.

Se muestra en las tablas el número de alumnos matriculados tanto en los grupos con docencia en español (DE) como en el grupo con docencia en inglés (DI), así como de este último grupo cuántos alumnos eran de procedencia extranjera y cuántos españoles. Además, se exponen los resultados académicos, diferenciando no sólo los grupos con DE con respecto al grupo con DI, sino también del grupo con DI el alumnado español del alumnado extranjero.

De la Tabla 3 correspondiente a la asignatura de Construcciones Históricas, se deduce que: el número de alumnos matriculados en el grupo con DI representa el $27 \%$ del total de alumnos matriculados en la asignatura, de los cuales sólo el $10 \%$ es alumnado con procedencia española (tal y como muestra también la tabla 2); prácticamente el mismo porcentaje (en torno al 5-6\%) de alumnos, tanto del grupo con DE como con DI, han obtenido una nota de notable, y de éste último grupo todos son extranjeros; el porcentaje de alumnos con una nota aprobada es mucho mayor en DI ( $70 \%$ del grupo, de los cuales el $85,7 \%$ son extranjeros) que en DE (el $55,5 \%$ del grupo); el porcentaje de alumnos suspensos, con respecto al total matriculados en la asignatura, en DI (10\%, todos extranjeros) es mucho más bajo que en DE (37\%); y por último, el porcentaje de alumnos 
no presentados es relativamente superior en DI ( $5 \%$ del grupo, de los cuales el $100 \%$ era extranjero) que en DE (3,7\% del grupo).

Tabla 3. Número de alumnos matriculados y resultados académicos obtenidos en la asignatura de Construcciones Históricas durante el curso 2018-2019

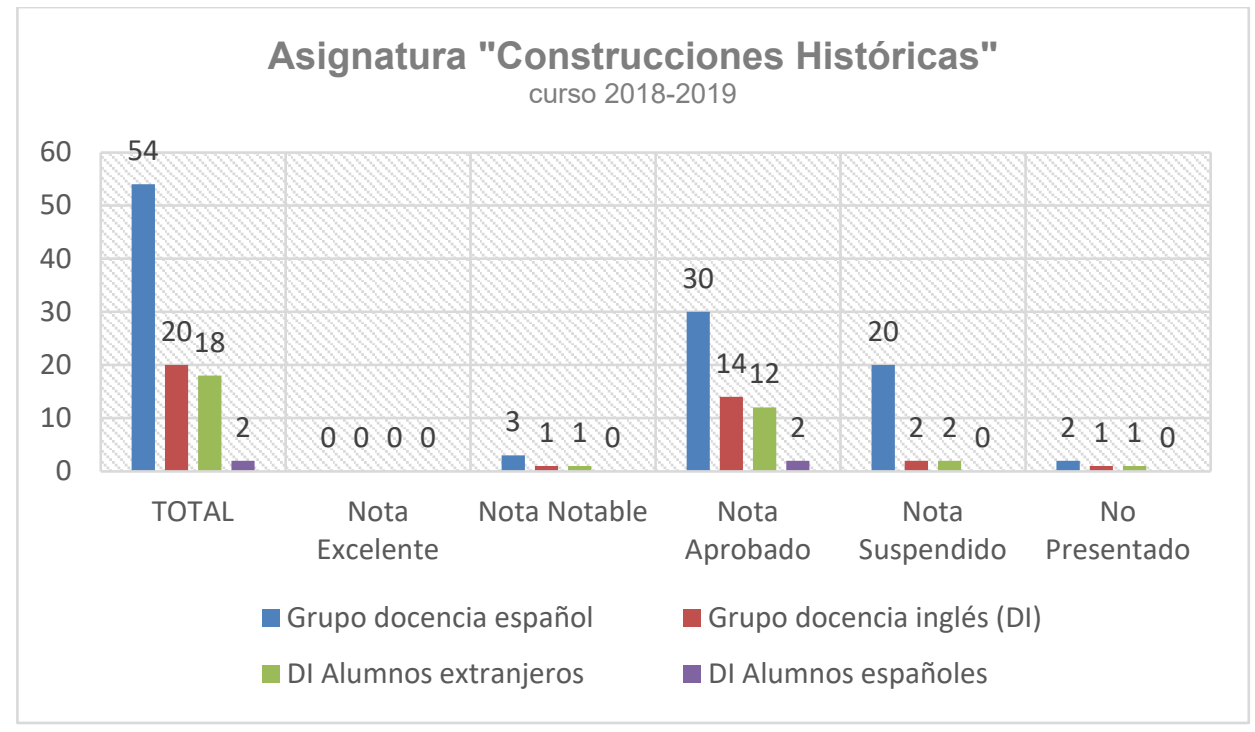

Tabla 4. Número de alumnos matriculados y resultados académicos obtenidos en la asignatura de Técnicas de Gestión presupuestaria durante el curso 2018-2019.

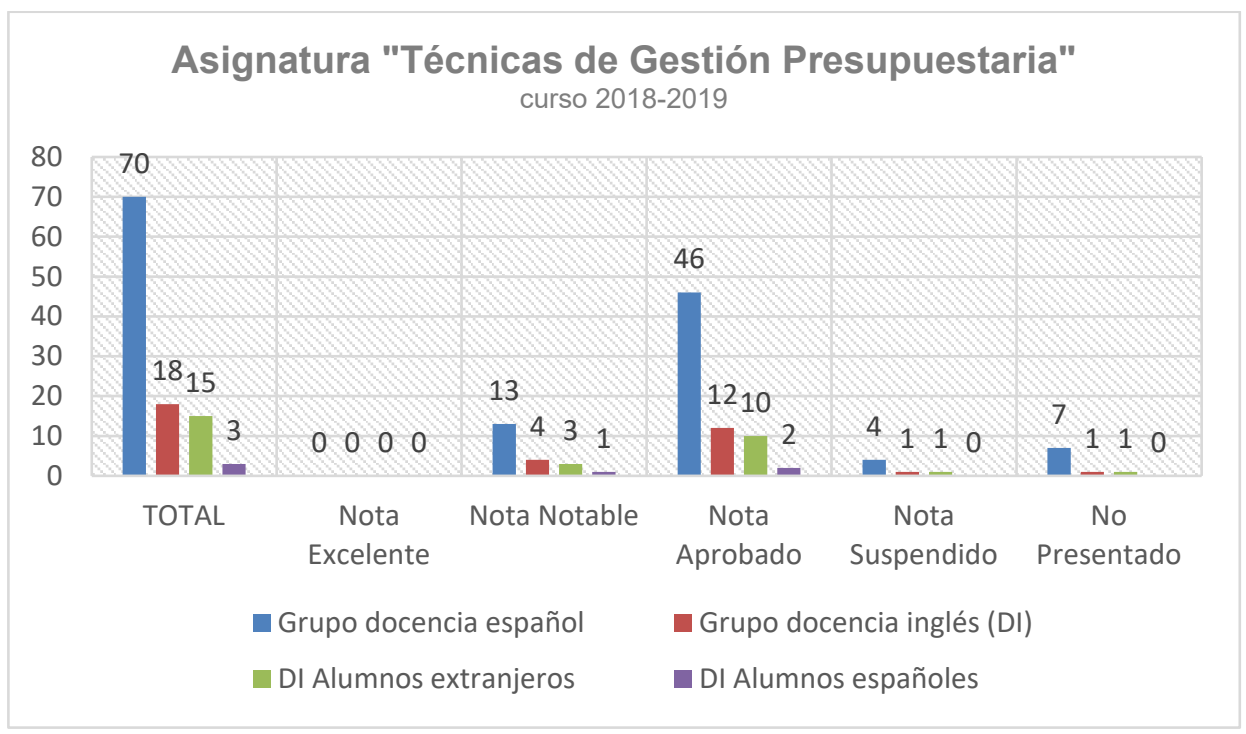


De la Tabla 4 correspondiente a la asignatura de Técnicas de Gestión Presupuestaria, se desprende que: el número de alumnos matriculados en el grupo con DI representa el 20,5\% del total de alumnos matriculados en la asignatura, de los cuales sólo el $17 \%$ es alumnado con procedencia española (tal y como muestra también la tabla 2); han obtenido una nota de notable un porcentaje relativamente mayor de alumnos del grupo con DI $(22,2 \%$, de los cuales el $75 \%$ es extranjero) que el grupo con DE (18,6\%); el porcentaje de alumnos con una nota aprobada es prácticamente igual (en torno al 65\%) en DI (el 83,3\% son extranjeros); el porcentaje de alumnos suspensos, con respecto al total matriculados en la asignatura, podría decirse que es igual tanto en DE como en DI (5,6\%, todos extranjeros); y por último, el porcentaje de alumnos no presentados es superior en DE ( $10 \%$ del grupo) que en DI (5,6\% del grupo, de los cuales el $100 \%$ era extranjero).

\section{Conclusiones}

Se ha podido comprobar que el nivel de inglés por parte del alumnado de otras nacionalidades ha sido sobradamente suficiente. Sin embargo, en el caso del alumnado español no lo ha sido tanto. Aún así, hay que decir que se ha visto un esfuerzo añadido por parte de este último para suplir esta carencia.

De la comparativa en las experiencias entre asignaturas impartidas y años académicos mostrados en la tabla 2, se desprende que en el Grado en Arquitectura Técnica la demanda de alumnos procedentes de otros países es mucho mayor frente a la demanda de alumnos españoles, aunque en el primer año básicamente sólo había alumnos españoles.

La casi total desaparición de la línea de inglés en la Escuela Técnica Superior de Ingeniería de Edificación de la UPV ha supuesto un retroceso en cuanto a la proyección internacional y calidad del Grado conseguidos durante los años en los que estuvo vigente.

La ampliación del léxico español a través de la gran cantidad de terminología específica que aparece durante la titulación del Grado en Arquitectura Técnica ya de por sí presenta ciertas dificultades para el alumnado. Además, si a esto se le suma la necesidad de hacerlo en un idioma extranjero, la dificultad es aún mayor. Del mismo modo, hay algunos casos con elevada complejidad de comprensión de la materia, debido al lenguaje tan concreto y completamente diferente del área de conocimiento, como es el caso de la asignatura de Tasaciones, Valoraciones y Peritaciones.

Del mismo modo, para facilitar y contribuir al aprendizaje del alumnado, se considera relevante la necesidad de disponer de un glosario o diccionario que desarrolle y contemple toda la terminología específica en español y su traducción a inglés, especialmente la concerniente a las asignaturas propias del módulo de Técnicas y Tecnologías de la Edificación. Esta bibliografía se podría hacerse extensiva también a las que corresponden a otros módulos, pero que igualmente pertenecen al mismo departamento.

La evolución del alumnado y los resultados de aprendizaje del grupo con DI han sido en general mejores que los grupos con DE en la mayoría de los casos. Lo cierto es que, 
incluso parece haber una mayor motivación e implicación por parte del alumnado. Así mismo, los buenos resultados académicos del grupo con DI han sido de alumnos de procedencia extranjera, tal y como muestran las tablas 3 y 4.

Se considera imprescindible y completamente justificada, para facilitar la comunicación con el estudiantado, la exigencia del nivel mínimo B2 de inglés no sólo al propio alumnado, sino también al profesorado. Sin embargo, y a pesar de que el profesorado tenga buen nivel de inglés, en ocasiones se presentan limitaciones por parte del alumno en la recepción de la información transmitida, bien debido al desconocimiento del idioma por no ser nativo, o bien por la materia objeto de estudio en sí misma, por ser completamente nueva y diferente de la formación recibida en los Grados que cursan en su lugar de origen.

Restablecer la línea de inglés en todo el Grado en Arquitectura Técnica en la Escuela Técnica Superior de Ingeniería de Edificación de la UPV, supondría recuperar no sólo ese nivel de calidad que le hace diferenciarse de otros centros nacionales, sino también convertirse en destino deseado por estudiantes de otros centros de ámbito internacional.

La baja demanda del alumnado español por la docencia en inglés, parece justificar la necesidad de promover entre este alumnado las ventajas académicas de recibir docencia en inglés en el Grado en Arquitectura Técnica. Se hace extensiva también la promoción hacia la faceta no curricular, ya que quizás pueda resultar incluso más atractiva.

La diversidad de nacionalidades y el moderado número de alumnos, reporta beneficios al grupo no sólo desde el punto de vista curricular por los resultados de aprendizaje, sino también desde la perspectiva no curricular. Ésta última debido el enriquecimiento personal, ya que el alumno prospera y aumenta sus habilidades personales, independientemente del Grado, por compartir docencia y trabajar con compañeros de otros países.

Es indispensable dotar de recursos al profesorado para la traducción del material docente, más aún si se desea dotar de cierto rigor y calidad al Grado. En este último caso, se hace necesaria la revisión terminológica por parte de traductores nativos con formación en la especialidad.

El prácticamente inexistente apoyo y ayuda para la traducción del material docente, ha implicado la realización de un importantísimo esfuerzo, que finalmente no ha sido reconocido ni valorado, resultando en definitiva desmotivador para el profesorado.

Destacar también que la dedicación académica del profesorado que imparte docencia en una lengua extranjera es mayor al que lo hace en la lengua de origen. De ahí la necesidad de dotar de reconocimiento docente al profesorado, como así lo muestran ciertas universidades españolas. Igualmente, y con el objetivo tanto de rentabilizar la labor desempeñada por el profesorado, como de mantener el estímulo de éste, se debe garantizar la continuidad de la docencia en inglés.

Por último, a pesar del sobreesfuerzo que ha supuesto y representa impartir la dociencia en inglés, y todo lo que ello conlleva, cabe decir que, como experiencia personal ha resultado una tarea muy interesante y enriquecedora. 


\section{Referencias}

Escuela Técnica Superior de Ingeniería de Edificación (s.f.-a). Convocatoria programa erasmus+ estudios. Curso 2021-2022. Anexo Khttps://www.etsie.upv.es/wp-content/uploads/2021/05/Anexo-IBases-especificas-ERASMUS-2021-2022.pdf> [Consulta: 16 de junio de 2021] [sin autoría reconocida]

Escuela Técnica Superior de Ingeniería de Edificación (s.f.-b). Erasmus 2021-2022. Programa de Intercambio para estudiantes ETSIE-UPVhttps://www.etsie.upv.es/internacional/estudiantesetsie/programas-de-intercambio-estudiantes-salientes/erasmus/> [Consulta: 16 de junio de 2021] [sin autoría reconocida]

Escuela Técnica Superior de Ingeniería de Edificación (s.f.-c)Estudiantes intercambio (Incomings). Asignaturas en inglés. <https://www.etsie.upv.es/wpcontent/uploads/2021/05/UPV_ETSIE_English_courses_2020-2021-r1.pdf> [Consulta: 16 de junio de 2021] [sin autoría reconocida]

Universitat Politècnica de València, Grado en Arquitectura Técnica (s.f.-a). Ficha informativa del Grado. <http://www.upv.es/titulaciones/GIE/indexc.html>. [Consulta: 16 de junio de 2021] [sin autoría reconocida]

Universitat Politècnica de València, Oficina de Programes Internacionals d'Intercanvi (s.f.-b). Convocatoria programa erasmust estudios. Curso 2020-2021. <http://www.upv.es/entidades/OPII/infoweb/pi/info/Anexo_I_20-21.pdf> [Consulta: 16 de junio de 2021] [sin autoría reconocida]

Universitat Politècnica de València, Servei d'Alumnat (s.f.-c). Información sobre la acreditacion del conocimiento de lenguas extranjeras, nivel B2, en los nuevos planes de estudio de grado. <https://www.upv.es/entidades/SA/ciclos/U0677392.pdf> [Consulta: 16 de junio de 2021] [sin autoría reconocida] 